\section{P394 SCHISTOSOMIASIS IN IRELAND, A CASE REPORT}

${ }^{1}$ Lubna Mahmood*, ${ }^{1}$ Abrar Haider, ${ }^{2}$ Wasim Asif. ${ }^{1}$ St. Luke's Hospital, Kilkenny, Ireland; ${ }^{2}$ St. Luke's Hospital , Kilkenny, Ireland

10.1136/archdischild-2019-epa.740

Introduction Schistosomiasis is a parasitic disease caused by flukes (trematodes) of genus Schistosoma . After malaria and intestinal helminthiasis, schistosomiasis is the third most devastating tropical disease in the world, being a major source of morbidity and mortality for developing countries in Africa, South America, Carribean , Middle East and Asia.

Presentation Nine years old boy presented to outpatients with history of intermittent haematuria for last seven months. Urine was bright red to pink in colour at the end of micturition. He recently visited his grandparents in Sudan where he had been swimming in river Nile. There was no history of trauma, no history of UTI.

Findings On examination, blood pressure was normal, he was circumcised. Systemic examination was unremarkable.

FBC, U\&E, LFTS, C3, C4, ASO titre and throat swab were normal, urine protein to creatinine ratio was normal however he has intermittent +1 blood on urine dipstick.

Urine microscopy showed 50 white cells and 60 red cells, urine and stool cultures were negative. Serology revealed Schistosoma ELISA positive at level 7 .

Abdominal X-ray did not show any renal tract calculus. Renal ultrasound showed thickened bladder wall of $6 \mathrm{~mm}$.

Treatment $\mathrm{He}$ was treated with PRAZIQUANTAL $40 \mathrm{mg} / \mathrm{kg}$ PO one dose only. One week after this treatment, his haematuria resolved and he had no further complaints.

Discussion Schistosomiasis is a disease caused by infection with parasitic blood flukes. These parasites live in certain types of freshwater snails. Individuals can become infected when skin comes in contact with contaminated water. The approach to diagnosis for returned travellers differs from the approach to diagnosis in endemic settings. Among returned travellers, serology is the most useful test, but it does not reflect definitive evidence of ongoing infection.

Diagnostic tools include direct assays (demonstration of eggs in the stool or urine via microscopy, or demonstration of antigen or DNA in the blood, urine, and/or stool) and indirect assays (demonstration of antibody in blood via serology).

Patients with schistosomiasis be treated promptly with Praziquantel in the presence or absence of clinical manifestations. A single dose of Praziquantel reduces the parasite burden substantially.

\section{P395 A CASE OF POTT'S PUFFY TUMOUR IN AN 11 YEAR OLD BOY}

Jonathan Clarke*, Megan Gilcreest, Ann Leahy. Cavan General Hospital, Cavan, Ireland

10.1136/archdischild-2019-epa.741

Case Report $\mathrm{CH}$ presented to Cavan General Hospital with a one day history of erythema and swelling over the right eye. This was associated with fever, lethargy and pain. $\mathrm{CH}$ was unable to open his right eye fully. There was normal visual acuity and eye movements. Diplopia was not a feature. The initial differential was periorbital cellulitis.

CT brain was performed and showed no evidence of intracranial pathology and no suspicious bone lesions. Pansinusitis was shown. He had a WBC of 22.9 (neutrophils of 20.6) and a CRP of 110.

$\mathrm{CH}$ was started on antibiotics as per local guidelines (ceftriaxone and clindamycin).

On day 4 of admission $\mathrm{CH}$ developed diplopia on lateral gaze. A repeat CT showed increased inflammatory changes of the right maxillary and frontal sinuses, infraorbital soft tissue density under the superior orbital wall and proptosis suggestive of orbital cellulitis. It also showed subcutaneous soft tissue swelling suggestive of underlying abscess formation. MRSA was also grown on a nasal swab with Vancomycin started to cover.

An MRI brain was performed which showed soft tissue oedema around the right orbit, osteomyelitis of the right frontal bone, as well as small subperiosteal collections extending into the right medial orbit and an intracranial subperiosteal collection. These findings confirmed a diagnosis of Pott's Puffy tumour.

Treatment involved adding metronidazole and rifampicin. FESS (functional endoscopic sinus surgery) with drainage of the orbital abscess was performed in Temple Street Children's Hospital. IV antibiotics were eventually stopped one month from presentation and $\mathrm{CH}$ was discharged home on $\mathrm{PO}$ antibiotics for a further 3 weeks having made an excellent recovery.

Discussion Pott's Puffy tumour was first described by Dr Perival Pott in 1760. It is characterised by osteomyelitis of the frontal bone with associated subperiosteal abscess. This presents as a fluctuant, tender swelling over the forehead. It is a life threatening complication of infectious sinusitis.

Complications include intracranial extension with epidural abscess, subdural empyema, meningitis and cerebral abscess. Treatment is through surgical drainage and prolonged IV antibiotics.

\section{P396 KAWASAKI DISEASE AND GROUP A STREPTOCOCCAL INFECTION: A CASE REPORT}

Triantafyllia Tsaprouni*, Vasiliki Melikoki, Evridiki Vouloumanou. Tzaneio General Hospital, Pireus, Greece

\subsection{6/archdischild-2019-epa.742}

Backgroud Kawasaki disease (KD) is an acute multi- system vasculitis which primarily affects children. Although the etiology remains unknown, it seems that $\mathrm{KD}$ is a response to superantigens in genetically susceptible individuals. It was reported that treatment with intravenous immunoglobulin (IVIG) is empirically effective because it inhibits bacterial superantigen induced production of proinflammatory cytocines. Case presentation summary A six-year-old boy presented with a history of fever for 5 days. Based on a positive strep test and typical red rash he was diagnosed with scarlet fever and was under treatment with amoxicillin. His clinical examination revealed bilateral conjunctival infection without exudate, cracked lips, strawberry tongue, injection of pharyngeal mucosa, maculopapular rash and unilateral cervical lymphadenopathy. Investigations showed increased WBC count, ESR $=52$ $\mathrm{mm} / \mathrm{hr}, \mathrm{CRP}=143 \mathrm{mg} / \mathrm{lt}$ and elevated liver enzymes. The film array test for upper respiratory tract infection was negative. $\mathrm{He}$ fulfilled the criteria of $\mathrm{KD}$, so he was started on IVIG and acetylsalicylic acid. He required more than one dose of IVIG in order to demonstrate an effect. On the 13th day 